\title{
EFICÁCIA DE GESTÃO DA RESERVA BIOLÓGICA DAS ARAUCÁRIAS, PARANÁ
}

\section{MANAGEMENT EFFECTIVENESS OF THE BIOLOGICAL RESERVE OF ARAUCARIA, PARANA}

\author{
Giulliano de Oliveira CAPOTE ${ }^{1}$, Isonel Sandino MENEGUZZO ${ }^{2 *}$, Juliane Coimbra \\ Bczuska $^{1}$, Saimon Francisco dos Santos BACH ${ }^{1}$ \\ ${ }^{1}$ Bachareis em Ciências Biológicas. Universidade Estadual de Ponta Grossa (UEPG), \\ Avenida Carlos Cavalcanti, 4748, Uvaranas, CEP 84030-900, Ponta Grossa, Paraná, Brasil. \\ $2^{2}$ Autor para contato: Professor Adjunto do Departamento de Geociências e do Programa \\ de Pós-Graduação em Geografia, Universidade Estadual de Ponta Grossa (UEPG), Avenida \\ Carlos Cavalcanti, 4748, Uvaranas, CEP 84030-900, Ponta Grossa, Paraná, Brasil. E-mail: \\ meneguzzo@uepg.br
}

\section{RESUMO}

O presente estudo teve como objetivo analisar a eficácia da gestão da Reserva Biológica das Araucárias, localizada no estado do Paraná. A metodologia utilizada envolveu a caracterização geral área de estudo, análise da legislação pertinente e aplicação de formulário de entrevista, na forma de questionário estruturado. Essa metodologia foi adaptada, abrangendo os seguintes âmbitos: Político-legal, Planejamento e Ordenamento, Administrativo, Conhecimento e Qualidade dos Recursos Protegidos, e estes divididos em critérios, cada qual com seu valor variando de zero a quatro, para dessa forma serem quantificados. Assim, chegou-se ao indicador de 52,94\% de eficácia de gestão na unidade de conservação ora pesquisada. Isso indica que a Reserva Biológica não possui uma gestão eficaz em todos os aspectos analisados, os quais deveriam ser efetivados de forma integral para atingir seu objetivo de criação, estabelecido em seu decreto de criação.

Palavras Chave: Efetividade, políticas ambientais, conservação da natureza.

\begin{abstract}
The present study aimed to analyze the effectiveness of the management of Araucaria Biological Reserve, located in the Parana state. The methodology involved the general caracterization of study area, review of relevant legislation and applying interview form, as questionnaire. This methodology has been adapted, covering the following areas: Political-legal, Planning and Management, Administration, Knowledge and Quality of Protected Resources, and these divided into criteria, each with a value ranging from zero to four, to be quantified. Thus it becomes the indicator of 52,94\% of effectiveness protected area management prays searched. This indicate that the Biological Reserve doesn't have effective management in all analyzed aspects, which should be effected in an integral way to achieve your objective of creating established in its creation decree.
\end{abstract}

Keywords: Effectivness. environmental policies. nature conservation. 


\section{INTRODUÇÃO}

No Brasil, desde pelo menos a década de 1960, existem áreas protegidas na modalidade de Reservas Biológicas (Pádua, 2015) com a finalidade de conservação ambiental.

De acordo com o Sistema Nacional de Unidades de Conservação (SNUC) uma Reserva Biológica (REBIO) se enquadra no grupo de Unidade de Proteção Integral e apresenta os seguintes objetivos (BRASIL, 2000):

A Reserva Biológica tem como objetivo a preservação integral da biota e demais atributos naturais existentes em seus limites, sem interferência humana direta ou modificações ambientais, excetuando-se as medidas de recuperação de seus ecossistemas alterados e as ações de manejo necessárias para recuperar e preservar o equilíbrio natural, a diversidade biológica e os processos ecológicos naturais.

$\S 1^{\circ} \mathrm{A}$ Reserva Biológica é de posse e domínio públicos, sendo que as áreas particulares incluídas em seus limites serão desapropriadas, de acordo com o que dispõe a lei.

$\S 2^{\circ}$ É proibida a visitação pública, exceto aquela com objetivo educacional, de acordo com regulamento específico.

$\S 3^{\circ}$ A pesquisa científica depende de autorização prévia do órgão responsável pela administração da unidade e está sujeita a condições e restrições por este estabelecidas, bem como àquelas previstas em regulamento (BRASIL, 2000).

Segundo o decreto de criação da REBIO das Araucárias, publicado em 23 de março de 2006, o documento estabelece como objetivos a preservação dos ambientes naturais com destaque para os remanescentes de Floresta Ombrófila Mista e Várzeas, bem como propiciar pesquisas científicas e desenvolver atividades de educação ambiental (BRASIL, 2006).

A REBIO das Araucárias compreende um dos maiores remanescentes de floresta com Araucárias com potencial de conservação dentro do Bioma Mata Atlântica, com uma área de 14.919 hectares, situada em parte dos municípios de Teixeira Soares, Imbituva e Ipiranga no estado do Paraná (MMA, 2014).

Sob o aspecto fitogeográfico, situa-se na região denominada de Campos Gerais do Paraná, com predomínio de vegetação composta por gramíneas, com a ocorrência de cerrados e matas ciliares ao longo dos cursos fluviais e de capões isolados (MAACK, 1950) com a existência do pinheiro Araucária (Araucaria angustifolia) (Bertol.) Kuntze). A região, mesmo tendo importantes remanescentes vegetacionais, apresenta significativas alterações antrópicas que propiciaram a existência de diversos impactos ambientais na paisagem (MENEGUZZO; ALBUQUERQUE, 2009).

Pesquisas indicam que os fragmentos florestais inseridos na REBIO das Araucárias funcionam como refúgio para diversos representantes da fauna, pois a maioria das áreas situadas no entorno foi transformada em agricultura, reflorestamento (Pinus sp. e Eucalyptus sp.) e pastagem para a criação de gado (BASTIANI et al., 2014).

Estudos envolvendo a eficácia de gestão de unidades de conservação (UCs) são de extrema relevância, pois podem permitir a identificação de problemas de diferentes ordens, que comprometem a conservação ambiental desses espaços legalmente instituídos. Assim, o poder público, no caso, o Instituto Chico Mendes de Conservação da Biodiversidade (ICMBio) tem a possibilidade de realizar procedimentos técnico-operacionais com o objetivo de minimizar e/ou solucionar problemas referentes à gestão de UCs.

Diante deste contexto, o presente artigo busca demonstrar a eficácia de gestão da REBIO das Araucárias, através da metodologia apresentada por Faria (2004) adaptada para o caso específico da UCs acima referida.

\section{MATERIAL E MÉTODO}

\section{Área de estudo}

A área de estudo situa-se na porção centro-leste do estado do Paraná. A UC localiza-se entre três municípios paranaenses. São eles: Imbituva, Teixeira Soares e Ipiranga (Figura 1). 
Figura 1: Cartograma de localização da REBIO das Araucárias no estado do Paraná, Brasil

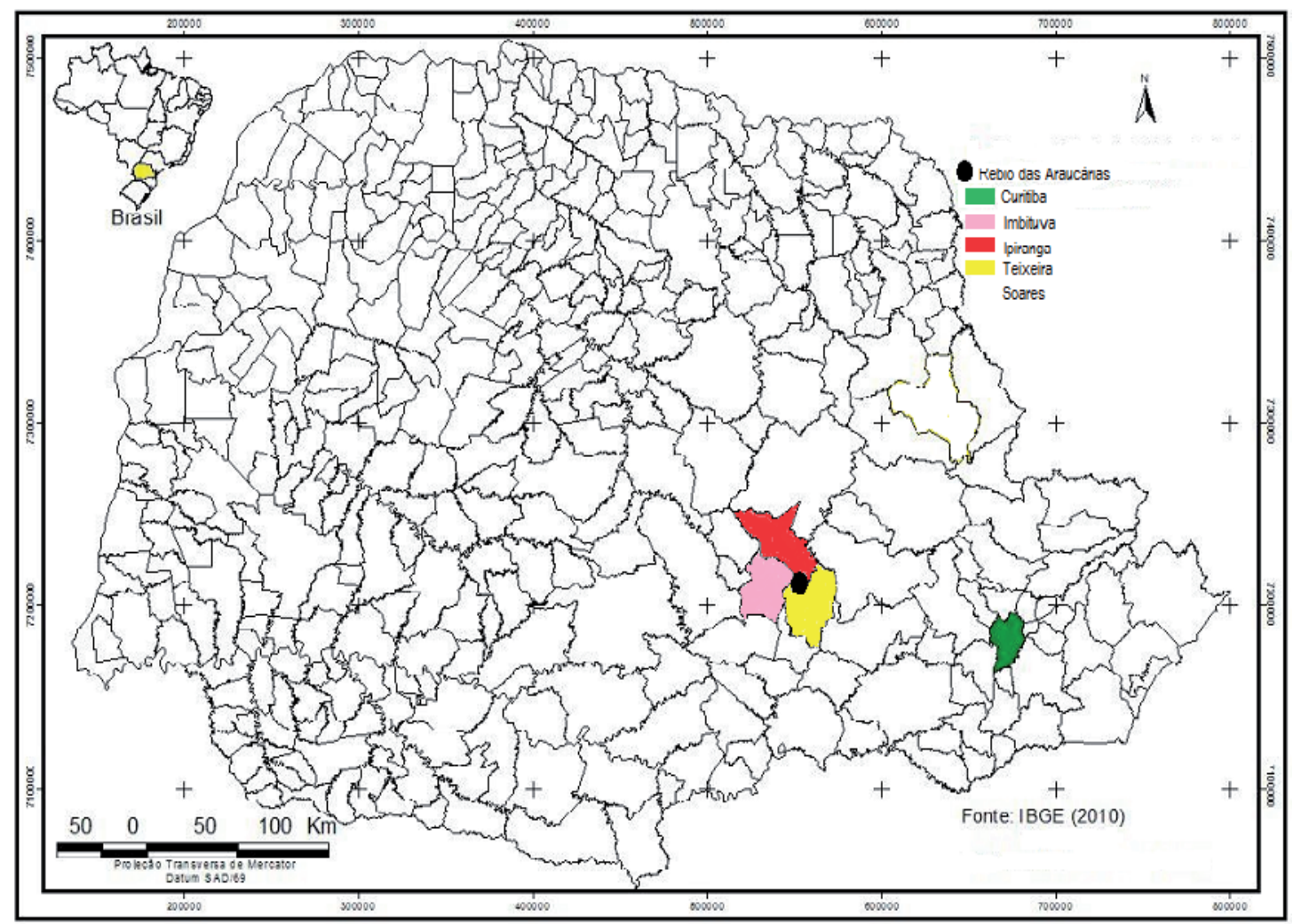

\section{Método}

Inicialmente, o estudo foi realizado mediante revisão bibliográfica (BASTIANI, et al. 2014; MAACK, 1950; MMA, 2014) e levantamento da legislação pertinente à REBIO das Araucárias. Outro procedimento adotado foi a aplicação de um formulário de entrevista (Cf. Quadro 1) junto ao gestor da unidade, com o objetivo de coletar os dados referentes à eficácia de gestão da área objeto de estudo.

Para a verificação da eficácia de gestão foi utilizada a metodologia já aplicada por Faria (2004) em UCs do estado de São Paulo. Ressalta-se que foram feitas adaptações/modificações dessa metodologia com vistas a adequá-la à legislação ambiental atualmente vigente no Brasil.

Nesse sentido, o estudo envolvendo a eficiência de gestão se deu em cinco âmbitos: Planejamento e Ordenamento, Administrativo, Político-Legal, Conhecimento e Qualidade dos Recursos Protegidos (Quadro 1). Foram avaliados 34 critérios, que visam à gestão da unidade em um cenário ótimo.
Quadro 1: Âmbitos e critérios abordados no questionário utilizado na análise da eficácia de gestão da REBIO das Araucárias (Adaptado de FARIA, 2004).

continua

\begin{tabular}{|l|l|}
\hline \multirow{4}{*}{ Âmbitos } & Critérios \\
\hline \multirow{5}{*}{$\begin{array}{l}\text { Planejamento e } \\
\text { Ordenamento }\end{array}$} & Existência e atualidade do plano de manejo \\
\cline { 2 - 3 } & Equipe de planejamento \\
\cline { 2 - 3 } & $\begin{array}{l}\text { Zoneamento } \\
\text { Compatibilidade dos usos com os objetivos da } \\
\text { unidade }\end{array}$ \\
\cline { 2 - 3 } & Programa de educação ambiental \\
\cline { 2 - 3 } & Execução do programa de educação ambiental \\
\hline \multirow{5}{*}{ Administrativo } & Formação do administrador \\
\cline { 2 - 3 } & Corpo de funcionários \\
\cline { 2 - 3 } & Recursos e financiamento \\
\cline { 2 - 3 } & Entrega dos recursos \\
\cline { 2 - 3 } & Organização dos arquivos referentes à unidade \\
\cline { 2 - 3 } & Regularização das atividades administrativas \\
\hline & Infraestrutura existente \\
\hline & Equipamentos e materiais da unidade \\
\hline
\end{tabular}


conclusão

Quadro 1: Âmbitos e critérios abordados no questionário utilizado na análise da eficácia de gestão da REBIO das Araucárias (Adaptado de FARIA, 2004).

\begin{tabular}{|c|c|}
\hline Âmbitos & Critérios \\
\hline \multirow{9}{*}{ Político-legal } & Instrumento legal de criação \\
\hline & $\begin{array}{l}\text { Cumprimento das políticas ambientais } \\
\text { vigentes }\end{array}$ \\
\hline & $\begin{array}{l}\text { Estabelecimento do Plano Nacional de Áreas } \\
\text { Protegidas }\end{array}$ \\
\hline & Situação fundiária \\
\hline & Apoio e participação comunitária \\
\hline & Apoio e/ou relacionamento interinstitucional \\
\hline & Plano de carreira dos funcionários \\
\hline & Programa de capacitação \\
\hline & Apoio e/ou facilitação interinstitucional \\
\hline \multirow{6}{*}{ Conhecimento } & Informações biofísicas \\
\hline & $\begin{array}{l}\text { Informações cartográficas sobre os recursos } \\
\text { naturais }\end{array}$ \\
\hline & $\begin{array}{l}\text { Informações socioeconômicas da unidade e } \\
\text { entorno }\end{array}$ \\
\hline & $\begin{array}{l}\text { Disponibilidade e conhecimento das normas } \\
\text { jurídicas }\end{array}$ \\
\hline & Pesquisas e projetos \\
\hline & $\begin{array}{l}\text { Acompanhamento de fenômenos naturais, } \\
\text { sociais e administrativos }\end{array}$ \\
\hline \multirow{4}{*}{$\begin{array}{l}\text { Qualidade } \\
\text { dos Recursos } \\
\text { Protegidos }\end{array}$} & Tamanho da unidade \\
\hline & $\begin{array}{l}\text { Forma aproximada da área e fragmentação da } \\
\text { unidade }\end{array}$ \\
\hline & Isolamento e insularidade \\
\hline & $\begin{array}{l}\text { Fatores naturais e antrópicos que afetam a } \\
\text { unidade }\end{array}$ \\
\hline
\end{tabular}

Os resultados do desempenho da gestão da UC foram obtidos realizando a somatória dos valores atribuídos pelo gestor, correspondendo à alternativa selecionada em cada critério, sendo este valor o "total observado" e por meio deste o cálculo da porcentagem dessa soma em relação à somatória máxima que poderia ser obtida, que seria o valor do "total máximo" onde a UC receberia o valor máximo quatro em todos os critérios. Os valores resultaram do confronto entre os totais máximos e totais observados referentes ao que foi respondido no questionário.

Esses valores foram tabulados em planilha eletrônica do software Excel, onde foram utilizadas as fórmulas adequadas para o cálculo dos totais, máximo e observado, os quais, quando comparados entre si, geram os valores percentuais em cada âmbito e no total da unidade.
Os percentuais obtidos foram confrontados com uma escala apropriada de avaliação de eficácia de gestão de UCs presente e adaptada do trabalho de Faria (2004), permitindo assim a classificação da qualidade da gestão da UC bem como de seus objetivos. A escala utilizada no procedimento de análise dos dados de eficácia de gestão possibilita uma avaliação normalizada para a UC.

A cada critério foi atribuído um valor, para associação deste a uma escala padrão de avaliação de eficácia de gestão de UCs. De acordo com a pontuação obtida, a UC pode se enquadrar em uma condição ideal ou representar uma condição de adversidade entrando assim em conflito com os objetivos de manejo da unidade.

A seguir é apresentado um quadro exemplificando os valores atribuídos a cada critério.

Quadro 2: Questão utilizada no questionário, dentro do critério abordado "situação fundiária", no âmbito Político-Legal

\begin{tabular}{|l|c|}
\hline Critério - Situação Fundiária \\
\hline $\begin{array}{l}\text { Questão 19.) Qual a situação fundiária da } \\
\text { UC? }\end{array}$ & Pontuação \\
\hline $\begin{array}{l}\geq 90 \% \text { da área declarada está sob domínio da } \\
\text { instituição. }\end{array}$ & 4 \\
\hline $\begin{array}{l}76 \%-89 \% \text { da área declarada está sob domínio } \\
\text { da instituição. }\end{array}$ & 3 \\
\hline $\begin{array}{l}51 \%-75 \% \text { da área declarada está sob domínio } \\
\text { da instituição. }\end{array}$ & 2 \\
\hline $\begin{array}{l}36 \%-50 \% \text { da área declarada está sob domínio } \\
\text { da instituição. }\end{array}$ & 1 \\
\hline $\begin{array}{l}\leq 35 \% \text { da área declarada está sob domínio da } \\
\text { instituição. }\end{array}$ & 0 \\
\hline
\end{tabular}

\section{RESULTADOS}

Dentro do âmbito Planejamento e Ordenamento, os critérios abordados procuram demonstrar a existência de instrumentos de planejamento que proporcionem diretrizes sólidas para que se alcancem os objetivos programáticos e mecanismos e meios que exerçam a função de ordenar as diversas atividades desenvolvidas (FARIA, 2004). As respostas apresentadas, nas questões 1 a 7 foram:

Questão 1. Em relação à existência da atualidade de um plano de manejo, assinale a alternativa que melhor se enquadra ao contexto da UC. Resposta: Não há plano de manejo, porém há perspectiva de elaboração. Valor 1. 
Questão 2. Quais são as características da equipe de planejamento? Resposta: Equipe interdisciplinar. Valor 3.

Questão 3. Sobre o zoneamento assinale a alternativa que melhor se enquadra ao contexto da UC. Resposta: A UC ainda não possui zoneamento. Valor 0.

Questão 4. Em que estágio se encontra o nível de planejamento da UC? Resposta: A área possui outros instrumentos de gestão. Valor 1.

Questão 5. Com relação à compatibilidade dos usos com os objetivos da unidade, assinale a alternativa que melhor se enquadra ao contexto da UC. Resposta: Usos incompatíveis e de incidência reduzida sobre os recursos protegidos. Valor 2.

Questão 6. Quanto a algum programa de educação ambiental na UC, assinale a alternativa que melhor se enquadra ao contexto da UC. Resposta: O programa está bem estruturado, abarca todas as ações e atividades para atingir seus objetivos específicos no intuito de alcançar os objetivos da unidade e as atividades desenvolvem-se normalmente. Valor 4.

Questão 7. Em relação à execução do programa de educação ambiental, assinale a alternativa que melhor se enquadra ao contexto da UC. Resposta: $\geq 90 \%$ das atividades planejadas têm sido executadas. Valor 4.

O âmbito Administrativo abarca os critérios que estão relacionados à capacidade institucional para gerir os recursos protegidos, dependendo diretamente das condições objetivas e dos instrumentos que a área dispõe para conduzir a aplicação das políticas e metas estabelecidas para a UC (FARIA, 2004). As respostas das questões 8 a 15 foram:

Questão 8. Qual o nível de formação do administrador da UC? Resposta: Universitário com cursos de especialização, iniciativa média/alta, presença média a alta nos sítios de trabalho. Valor 3.

Questão 9. Com relação ao corpo de funcionários, a quantidade atual é: Resposta: $36-50 \%$ do ótimo. Valor 1.

Questão 10. A respeito dos recursos/ financiamento da UC, assinale a alternativa que melhor se enquadra. Resposta: A área recebe $35 \%$ ou menos do ótimo. Valor 0.

Questão 11. Como se dá a regularidade de entrega dos recursos? Resposta: Há entrega com alguma regularidade, com variações previsíveis. Valor 2.

Questão 12. Como se encontram organizados os arquivos referentes à UC? Resposta: Os arquivos são simples, mas suficientemente completos para proporcionar um bom suporte à administração. Valor 3 .

Questão 13. Em relação à regularização das atividades administrativas, assinale a alternativa que melhor se enquadra ao contexto da UC. Resposta: O sistema não apresenta integração de todas as atividades, porém é satisfatório em vista da flexibilidade e controle do sistema sobre as principais atividades administrativas. Valor 3.

Questão 14. A infraestrutura existente está adequada à demanda atual e se aplica aos programas e/ ou atividades desenvolvidas? Resposta: As estruturas existentes não são suficientes em quantidade, mas possuem qualidade satisfatória que permitem atender a maioria das atividades da UC. Valor 3.

Questão 15. A respeito dos equipamentos e materiais da UC, estes se encontram em condições adequadas para uso? Resposta: Possui transporte e comunicação em boas condições e parte dos demais equipamentos e materiais necessários. Valor 3.

O âmbito Político-legal procura averiguar a existência de uma sólida base que realize a gestão e se as unidades correspondem a essas políticas ditadas ou adotadas pelo gestor (FARIA, 2004). As respostas para as questões 16 a 24 foram as seguintes:

Questão 16. Em relação ao instrumento legal de criação da UC, qual a alternativa que melhor se enquadra ao contexto atual? Resposta: O nível do instrumento jurídico de criação da área é satisfatório e encontra-se regulamentado, porém o mesmo necessita adequar-se aos conceitos teóricos sobre o assunto e à realidade política nacional e regional. Valor 3.

Questão 17. Sobre o cumprimento das políticas ambientais vigentes (SNUC e seu Decreto Regulamentador) atualmente tem-se: Resposta: $36 \%$ - $50 \%$ do que as políticas ambientais preconizam é cumprido. Valor 1.

Questão 18. No tocante ao que é estabelecido pelo Plano Nacional de Áreas Protegidas atualmente tem-se: Resposta: Não foi respondida pelos gestores da UC, pois no período em que foi realizada a entrevista, não possuíam conhecimento do Plano Nacional de Áreas Protegidas. Assim, foi atribuído o valor 0 como resposta.

Questão 19. Qual a situação fundiária da UC? Resposta: $\leq 35 \%$ do que é preconizado é efetivado. Valor 0.

Questão 20. Como é o apoio e participação co- 
munitária em relação à UC? Resposta: Não há cooperação entre a administração e comunidade, porém os vizinhos reconhecem parcialmente o valor intrínseco da área; os benefícios são poucos ainda. Valor 1.

Questão 21. Como se dá o apoio e/ou o relacionamento interinstitucional do ICMBio? Resposta: A definido, e existência de B com maior intensidade que C. Sendo A referente à jurisdição e papel institucional, $\mathrm{B}$ coordenação com outros órgãos e $\mathrm{C}$ o intercâmbio de informações, experiências e recursos. Valor 3.

Questão 22. Sobre o plano de carreira dos funcionários do ICMBio, assinale a alternativa que melhor se enquadra ao seu contexto. Resposta: Os funcionários contam com planos de carreira com progressão gradual que estimula a permanência no serviço e proporciona ânimo para o trabalho. Valor 4.

Questão 23. Como é o programa de capacitação dos funcionários? Resposta: Há um programa de capacitação organizado que é cumprido eficientemente para a especialização dos funcionários. Valor 4.

Questão 24. Como se dá o apoio e/ou facilitação intra-institucional? Resposta: A estrutura atual da instituição não lhe permite dar assistência estável para as atividades desenvolvidas na unidade. Valor 2.

No âmbito Conhecimento, o enfoque é a necessidade permanente dos diretores da UC terem informações de qualidade disponíveis e que estas possam ser utilizadas e disseminadas, sendo estas importantes elementos para a compreensão da complexidade e fragilidade dos recursos protegidos e seu manejo inteligente (FARIA, 2004). Este âmbito compreendeu as questões 25 a 30 . As respostas foram:

Questão 25. Sobre as informações biofísicas, assinale a alternativa que melhor se enquadra ao seu contexto: Resposta: A área está implementando estudos visando obter as informações necessárias. Valor 2.

Questão 26. Como se encontram as informações cartográficas sobre os recursos naturais protegidos na UC? Resposta: A área está implementando estudos visando obter as informações necessárias. Valor 2.

Questão 27. Sobre as informações socioeconômicas da UC e de seu entorno, assinale a alternativa que melhor se enquadra ao seu contexto. Resposta: A área está implementando estudos visando obter as informações necessárias. Valor 2.

Questão 28. Em relação à disponibilidade e ao conhecimento externo e interno sobre as principais normas jurídicas incidentes na gestão e manejo da
UC, assinale a alternativa que melhor se enquadra ao contexto atual. Resposta: Moderada disponibilidade e pouca difusão. Valor 2.

Questão 29. Sobre as pesquisas e projetos referentes à UC, assinale a alternativa que melhor se enquadra ao seu contexto. Resposta: Os conhecimentos gerados estão parcialmente disponíveis em cadastros locais e são utilizados para retroalimentar o manejo. Valor 3.

Questão 30. Em relação à capacidade da UC de implementar sistemas de acompanhamentos de fenômenos naturais, sociais e administrativos que permitam identificar mudanças e tomar decisões adequadas a essas mudanças, assinale a alternativa que melhor se enquadra ao seu contexto. Resposta: A unidade conta com alguns instrumentos para o monitoramento e retroalimentação, que atendem parcialmente necessidades básicas do manejo. Valor 2.

Dentro do âmbito Qualidade dos Recursos Protegidos são avaliados fatores que podem ser determinantes para o cumprimento dos objetivos de manejo das unidades de proteção integral (FARIA, 2004). Abrangeu as questões 31 a 34, e obtiveram-se as seguintes respostas:

Questão 31. O tamanho da UC é condizente com seus objetivos de criação?

Resposta: A área tem entre $51 \%$ e $75 \%$ da superfície total ótima. Valor 2.

Questão 32. Sobre a forma aproximada da área e a condição de fragmentação da totalidade da área, assinale a alternativa que melhor se enquadra ao contexto da UC. Resposta: Forma muito irregular, fragmentada. Valor 0.

Questão 33. Em relação ao isolamento ou insularidade, assinale a alternativa que melhor se enquadra ao contexto da UC. Resposta: Áreas silvestres contíguas, podendo ser da mesma ou de outras unidades, ou mesmo áreas naturais privadas. Valor 4.

Questão 34. Sobre os fatores naturais e antrópicos que afetam a estabilidade ambiental, assinale a alternativa que melhor se enquadra ao contexto da UC. Resposta: Fatores cujos efeitos são graves, porém são reconhecidos como manejáveis, evitáveis ou de fácil recuperação. Valor 2.

Após a aplicação da metodologia pôde-se verificar que a REBIO das Araucárias obteve um percentual de 52,94\% de eficácia em sua gestão. Esse valor, de acordo com o sistema de classificação de eficácia, 
apresenta "padrão inferior" (41 - 54,99\%), onde são considerados existentes os recursos para a gestão da unidade, porém a área se encontra vulnerável a fatores externos por haver somente os meios e recursos mínimos necessários para a gestão, podendo vir a acarretar o não cumprimento de alguns dos objetivos da unidade (FARIA, 2004).

Deve-se ressaltar que apesar do valor final obtido para a REBIO ser enquadrado como inferior, vários critérios receberam notas consideradas altas, remetendo ao fato de que estes são cumpridos de acordo com os objetivos e normas da UC, dentro da realidade possível e do alcance dos gestores, trabalhando com os recursos disponíveis para tal. Outros critérios, porém, obtiveram notas baixas ou medianas por encontrarem-se fora do alcance do gerente da unidade.

Assim, a REBIO das Araucárias obteve um resultado considerado baixo no âmbito da escala de avaliação de eficácia de gestão tornado evidente a necessidade de efetivação das políticas públicas ambientais vigentes.

\section{CONCLUSÕES}

Desde a sua criação em 2006, a REBIO das Araucárias ainda não vem sendo alvo de políticas ambientais efetivas. A morosidade em resolver os principais problemas (ausência de plano de manejo e de regularização fundiária, por exemplo) que afligem esta UC certamente comprometem a conservação de seus elementos naturais.

Com a aplicação da metodologia pôde-se verificar o valor de 52,94\% de eficiência de gestão para a REBIO das Araucárias. Dessa maneira ficou clara, a necessidade de implementação de medidas nos cinco âmbitos verificados, para que o parque cumpra efetivamente seus objetivos conforme consta em seu decreto de criação.

Cabe ao poder público federal realizar os procedimentos técnico-científicos e políticos de forma eficiente, para que a REBIO não conste apenas no papel e cumpra também sua função ambiental, educacional e científica, tal como estabelece seu decreto de criação e demais legislações que contemplam esta UC.

\section{QUESTÕES ÉTICAS}

O projeto de pesquisa foi submetido à Comissão de Ética em Pesquisa da Universidade Estadual de
Ponta Grossa/Plataforma Brasil - Parecer de aprovação número 572.617.

\section{REFERÊNCIAS BIBLIOGRÁFICAS}

BASTIANI, E. D. ; BAZILIO, S. ; PEREIRA, A. D. ; BCZUSKA, J. C. ; FARACO JUNIOR, J. H. ; MARQUES, J. L. A. Levantamento preliminar de mamíferos de médio e grande porte na Reserva Biológica das Araucárias no Estado do Paraná, Brasil. Gramado: $7^{\mathbf{0}}$ Congresso Brasileiro de Mastozoologia. 2014, p. 135-136.

BRASIL. Sistema Nacional de Unidades de Conservação. 2000. Disponível em: <www.planalto.gov.br/ccivil_03/leis/ L9985.htm>. Acesso em: 26 de março. 2014.

Decreto de 23 de março de 2006. Disponível em:< https://www.planalto.gov.br/ccivil 03/ Ato20042006/2006/Dnn/Dnn10795.htm>. Acesso em: $2 \overline{2}$ de out. de 2014.

FARIA, H. H. Eficácia de gestão de unidades de conservação gerenciadas pelo Instituto Florestal de São Paulo, Brasil. Presidente Prudente, 2004. 385 f. Tese (Doutorado em Geografia) - Universidade Estadual Paulista de Presidente Prudente, São Paulo.

MAACK, R. Mapa fitogeográfico do Estado do Paraná. Curitiba: Instituto de Biologia e Pesquisa Tecnológica e Instituto Nacional do Pinho, 1950. 85 x $120 \mathrm{~cm}$. Escala 1: 750.000 .

MENEGUZZO, I. S. ; ALBUQUERQUE, E. S. A política ambiental para a região dos Campos Gerais do Paraná. Ra'e Ga, Curitiba, n.18, p. 51-58, 2009.

MMA. Ministério do Meio Ambiente. Relatório Parametrizado - Unidade de Conservação: Reserva Biológica das Araucárias. 2014. Disponível em: < http://sistemas.mma.gov.br/cnuc/index. php?ido=relatorioparametrizado.exibeRelatorio\&relatorio Padrao $=$ true\&idUc $=276>$. Acesso em: 07 de dezembro de 2014.

PÁDUA, M. T. J. Conservando a natureza no Brasil. Curitiba: Fundação Grupo $\mathrm{O}$ Boticário de Proteção à Natureza, 2015. 